Мустафаев А.Г.

\title{
НЕЙРОСЕТЕВАЯ МОДЕЛЬ ПРОГНОЗИРОВАНИЯ УРОВНЯ СОЛНЕЧНОЙ ЭНЕРГИИ ДЛЯ ЗАДАЧ АЛЬТЕРНАТИВНОЙ ЭНЕРГЕТИКИ
}

\begin{abstract}
Аннотация: Одной из проблем сдерживающих развитие активного использования возобновляемых источников энергии является определение оптимального размещения ветровой или солнечной электростанции на земной поверхности. В данной работе разработана модель прогнозирования уровня солнечной энергии в регионе, позволяющая выбрать наиболее эффективное место для расположения солнечной электростанции. Прогноз об уровне солнечной энергии дается при помощи искусственной нейронной сети прямого распространения, обученной на основе данных метеорологических станций, при помощи алгоритма обратного распространения. Проектирование нейронной сети проводилось при помощи пакета Neural Network Toolbox из MATLAB 8.6 (R2015b). Сравнение результатов прогноза уровня солнечной энергии искусственной нейронной сети с актуальнымизначениями показывают хорошую коррелячию. Это подтверждает возможность использования искусственных нейронных сетей для моделирования и прогнозирования в регионах где отсутствуют данные об уровне солнечной энергии, но есть иные данные метеорологических станций
\end{abstract}

Ключевые слова: солнечная энергия, искусственная нейронная сеть, моделирование, многослойный персептрон, обратное распространение ошибки, прогнозирование, альтернативная энергетика, сеть прямого распространения, обучение с учителем, карта ресурсов

Abstract: One of the problems hampering the development of the active use of renewable energy is to determine the optimal placement of wind and solar power plants on the earth's surface. The paper presents a model for predicting the level of solar energy in the region allowing choosing the most effective location for solar power location. The forecast of the level of solar energy is given using an artificial neural network of direct distribution educated on the basis of meteorological stations data using the back-propagation algorithm. Designing a neural network was carried out with the Neural Network Toolbox package of MATLAB 8.6 (R2015b). Comparison of results of forecasting solar energy performed by artificial neural network level with the current values shows a good correlation. This confirms the possibility of using artificial neural networks for modeling and forecasting in regions where there are no data on the level of solar energy, but some other data of meteorological stations is present. 
Keywords: alternative power engineering, prediction, errorback propagation, multilayer perceptron, modeling, artificial neural network, solar energy, feedforward network, supervised learning, resource map

\section{Введение}

Солнечная энергетика представляет собой одно из перспективных направлений возобновляемой энергетики, основанное на непосредственном использовании солнечного излучения с целью получения энергии для отопления, электроснабжения и горячего водоснабжения. Актуальность солнечной энергетики постоянно растет, потому что солнечная энергия является экологически чистой. За 10 минут Земля получает больше энергии от Солнца, чем человечество производит за весь год.

В настоящее время карты ресурсов солнечной энергии созданы и широко доступны для определения потенциала определенной территории в солнечной энергии. Эти карты создаются на основе спутниковых снимков и данных метеорологических станций. Однако большой масштаб карт ресурсов, находящихся в свободном доступе, различия в микроклимате и топографии не позволяют принять решение о выборе оптимального проекта солнечной электростанции. Ошибка в выборе места расположения солнечной электростанции может существенно снизить возможное количество солнечных дней в году [1].

Для построения эффективных систем солнечной энергетики необходимы точные данные об солнечной освещенности поверхности земли. Традиционный способ мониторинга потока солнечной энергии заключается в размещении системы пиранометров на значительной территории и их обслуживания, что значительно увеличивает стоимость сбора данных.

По этой причине проблема разработки метода сбора информации о солнечной энергии, использующего климатические данные регистрируемые стационарными метеостанциями, является актуальной.

Целью настоящей работы является разработка нейросетевой модели прогнозирующей значение уровня солнечной энергии на основе косвенных данных метеорологических станций.

Искусственные нейронные сети - инструмент моделирования и прогнозирования, получивший широкое признание в качестве метода решения трудноформализуемых задач с независимыми параметрами. Нейронные сети обучаются на тестовых примерах, обладают высокой отказоустойчивостью в отношении зашумленных и неполных данных. Обученная нейронная сеть может быть использована для прогнозирования предметной области [2, 3].

Искусственная нейронная сеть является одним из подходов технологии создания интеллектуальных систем, основанных на имитации поведения человеческого мозга. Нейронные сети разделяются на две категории: прямого распространения (feed-forward) и обратного распространения (feedback). B feed-forward нейросетях сигнал распро- 
страняется только в одном направлении - от входов к выходам. B feedback нейросети выходной сигнал от нейрона передается на вход другого нейрона, на том же или предыдущем уровне. Чаще всего используются сети прямого распространения, в которых нейроны делятся на группы с общим выходным сигналом - слои, при этом на каждый нейрон первого слоя подаются элементы внешнего выходного сигнала, а все выходы нейронов $i$-го слоя подаются на каждый нейрон слоя $(i+1)$.

Многослойный персептрон - общий случай сети прямого распространения. Он содержит три типа слоев нейронов: входной, скрытый и выходной (рис. 1) [4]. Многослойный персептрон обладает высокой степенью связности, реализуемой посредством синаптических соединений. Каждый нейрон сети имеет гладкую нелинейную функцию активации. Многослойные нелинейные нейронные сети позволяют формировать более сложные связи между входами и выходами, чем однослойные линейные. Доказано, что трехслойная нейронная сеть с одним скрытым слоем может быть обучена аппроксимировать с произвольной точностью любую непрерывную функцию [5].

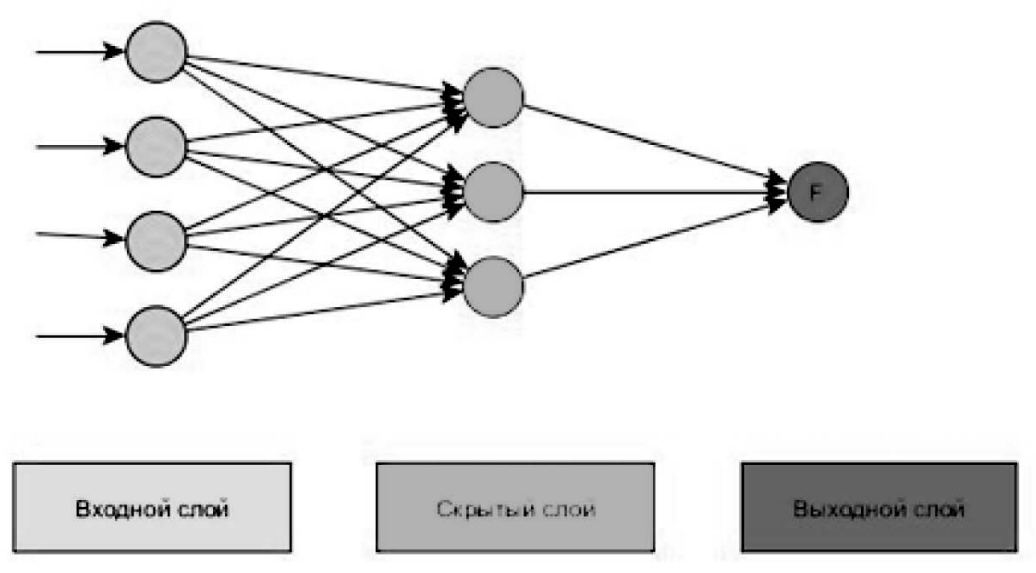

Рис. 1. Простейший многослойный персептрон

\section{Сбор данных}

Метеорологические данные о погодных условиях на пятнадцати метеостанциях Северо-Кавказского региона за период 2000- 2010 гг были получены с использованием технологии «Аисори» из единого государственного фонда данных ВНИИ гидрометеорологической информации (ВНИИГМИ-МЦД) [6]. Были учтены следующие географические и метеорологические параметры широта, долгота и высота над уровнем моря точки наблюдения, длительность светового дня, температура, относительная влажность, солнечная освещённость. Данные с семи метеостанций использовались для обучения нейронной сети (обучающая выборка), данные четырех метеостанций для тестирования (тестовая выборка), данные четырех метеостанций для проверки разработанной модели (валидационная выборка). 


\section{Разработка искусственной нейронной сети}

Разработка нейронной сети требует выполнения нескольких этапов (рис. 2).

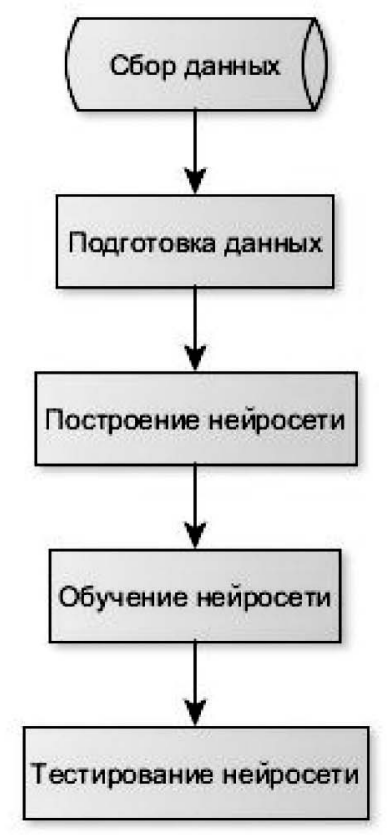

Рис. 2. Этапы разработки искусственной нейронной сети.

Подготовка данных заключается в их нормализации и дополнении отсутствующих данных (усреднением пограничных данных).

На этапе обучения нейронная сеть восстанавливает целевую функцию по множеству наборов обучающей выборки, т.е. решает задачу интерполяции. На этапе использования обученной нейронной сети (получении прогноза) она будет использовать восстановленную зависимость для получения прогнозируемой величины, т.е. решать задачу экстраполяции. Для корректного решения задачи экстраполяции как задачи интерполяции необходимо обеспечить стационарность временного ряда признаков, распределение значений ряда должно быть инвариантно относительно момента времени, для которого оно построено.

Для улучшения качества и скорости обучения нейронной сети необходимо преобразовать (нормализовать) входные данные к диапазону [-1,1]. Формула пересчета значения признака х для $i$-го примера выборки в интервал $[a, b]$ такова [7]:

$$
\tilde{x}_{i}=\frac{\left(x_{i}-x_{\min }\right)(b-a)}{\left(x_{\max }-x_{\min }\right)}+a
$$

где $x_{\min }, x_{\max }$ - минимальное и максимальное выборочные значения признака. 
Для проектирования нейронной сети был использован пакет Neural Network Toolbox из MATLAB 8.6 (R2015b), представляющий полный набор функций и структур данных, описывающих функции активации, алгоритмы обучения, установку синаптических весов, визуализацию и др. Последовательность стадий создания нейронной сети в MATLAB 8.6 (R2015b) показана на рис. 3.

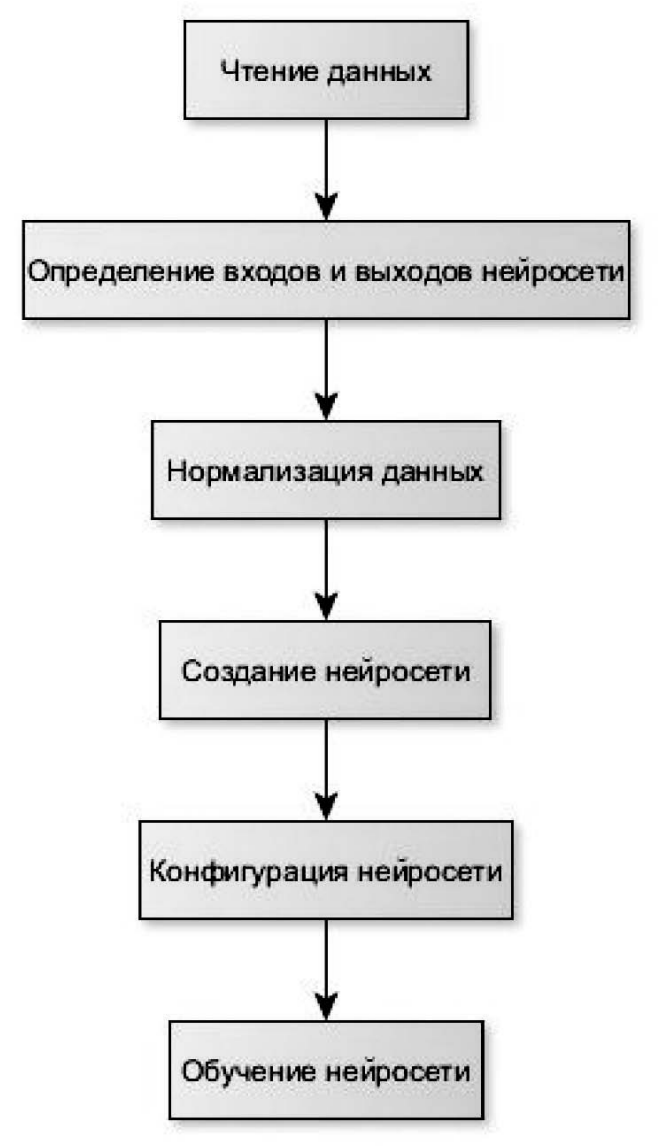

Рис. 3. Стадии создания нейронной сети в MATLAB

Для решения поставленной задачи была выбрана модель многослойного персептрона, имеющего три слоя: семь нейронов в первом слое, пять нейронов в скрытом слое и один нейрон в выходном слое (рис. 4). Входной слой нейронной сети выполняет распределительные функции. Нейроны скрытого слоя имеют пороговую функцию активации и именно они производят непосредственную обработку информации. Выходной слой необходим для обработки информации (определения порога ошибки) и выдачи результата. Для предотвращения эффекта «переобучения» нейронной сети размерность (количество нейронов) скрытого слоя должна быть ниже чем размерность обучающей выборки. Для активации скрытого и выходного слоя использовалась логистическая и линейная функции соответственно. 


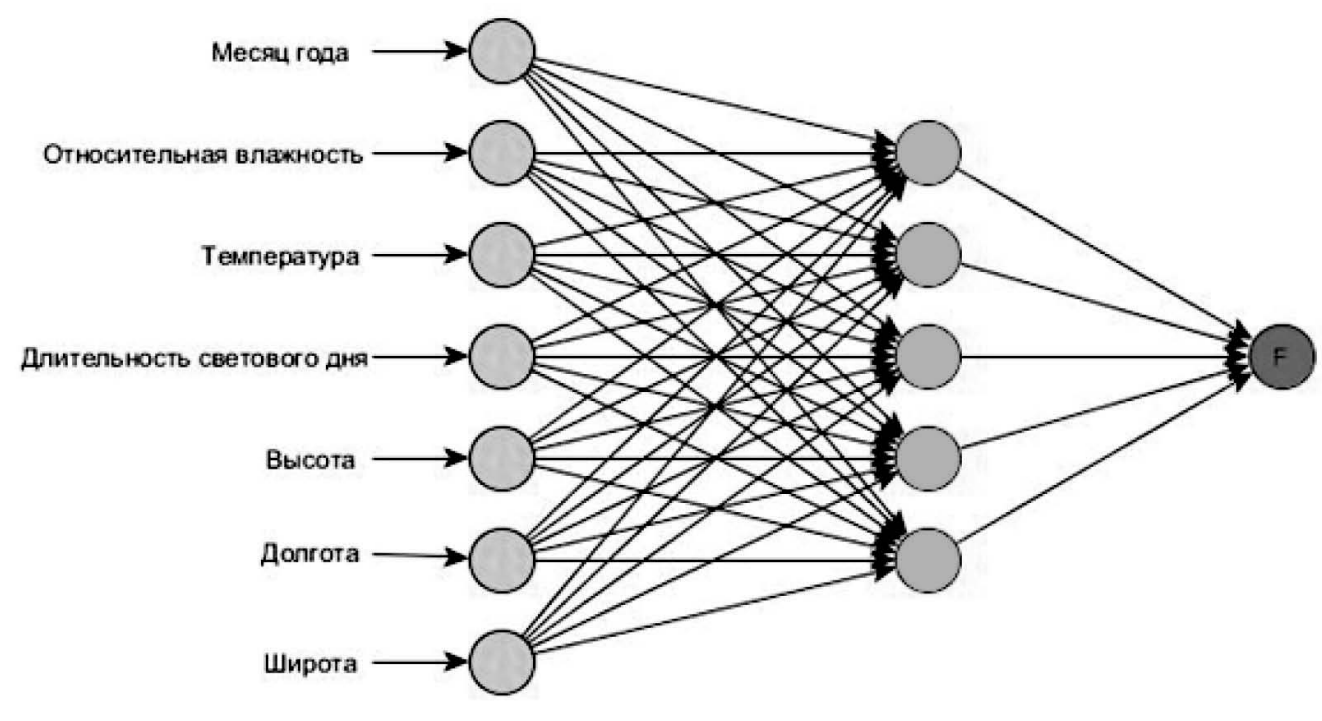

Входной елой
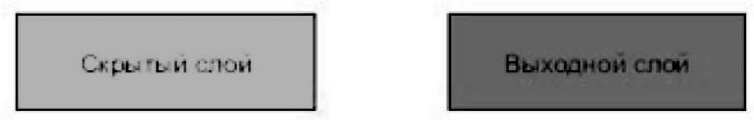

Рис. 4. Архитектура нейронной сети

Для построения нейронной сети с хорошей обобщающей способностью необходимо определить меру Вапника-Червоненкиса для данной топологии нейронной сети [8]:

$$
2\left[\frac{K}{2}\right] N \leq V C \operatorname{dim} \leq 2 N_{w}\left(1+\lg N_{n}\right)
$$

где $N$ - размерность данных на входе; $K$ - количество нейронов в скрытом слое; $N_{w}$ - общее количество весов сети; $N_{n}$ - общее количество нейронов сети.

Способ точного определения VCdim отсутствует и в качестве приближенного значения VCdim используется общее количество весов сети. Установлено [9], что хорошие показатели обобщения достигаются, когда количество обучающих примеров в несколько раз превышает количество весов сети.

Обучение сети производилось методом обратного распространения ошибки, общая схема которого описывается следующим образом:

1. Инициализировать синаптические веса нейронов маленькими случайными значениями;

2. Выбрать очередной обучающий вектор из обучающей выборки и подать его на вход сети;

3. Вычислить результат на выходе нейронной сети;

4. Определить разность между текущим выходом сети и требуемым выходом (целевым значением); 
5. Скорректировать синаптические веса нейронной сети для минимизации ошибки; 6. Повторять шаги с 2 по 5 для каждого вектора обучающей выборки до тех пор, пока ошибка на всем множестве не достигнет приемлемого уровня.

После того как нейронная сеть была обучена, она была использована для прогнозирования уровня солнечной энергии на основе валидационной выборки (рис. 5).

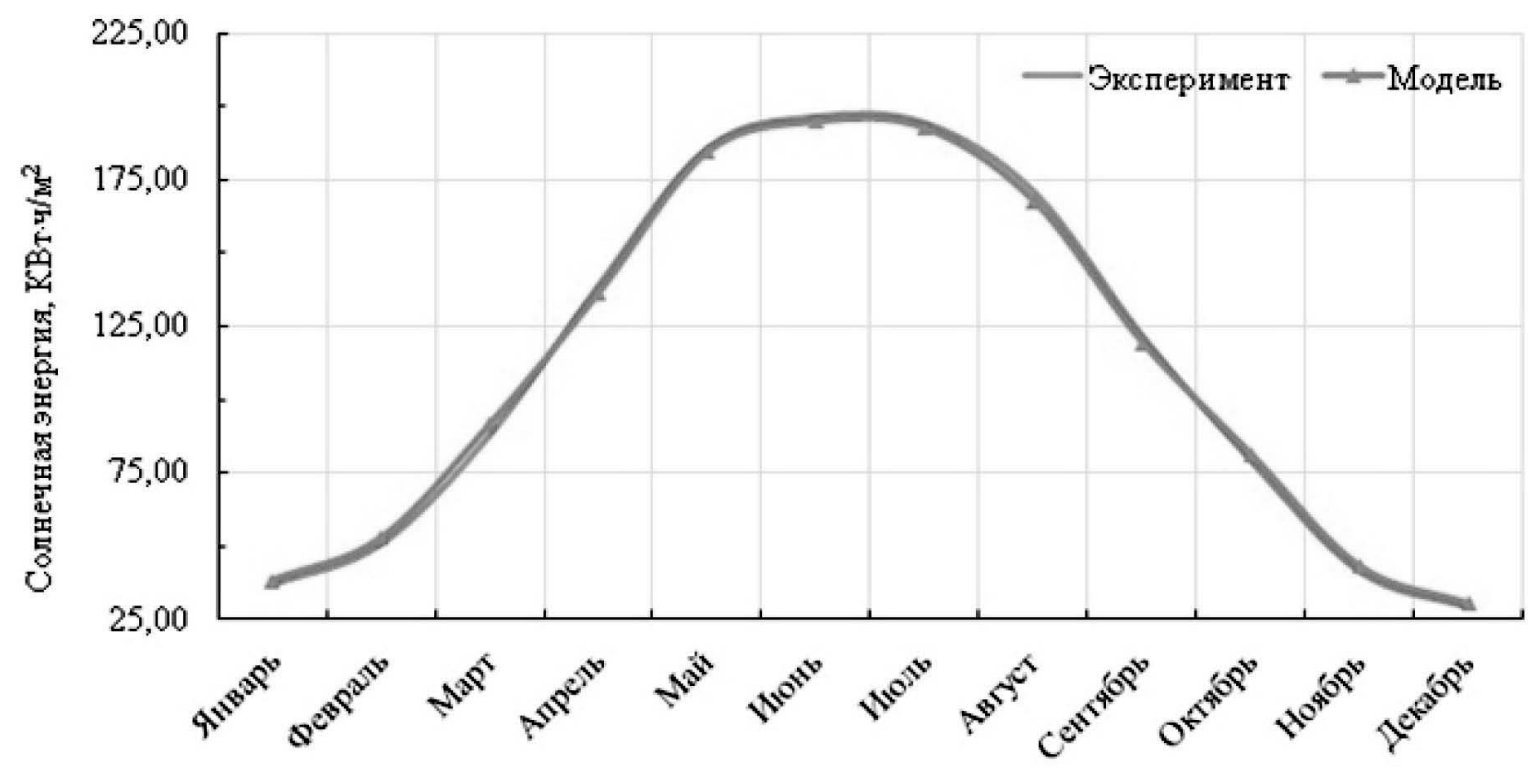

Рис. 5. Измеренные и прогнозные значения среднесуточной солнечной энергии в г.Махачкала

\section{Заключение}

Сравнение результатов прогноза уровня солнечной энергии данного искусственной нейронной сетью с актуальными значениями показывают хорошую корреляцию. Это подтверждает возможность использования искусственных нейронных сетей для моделирования и прогнозирования в регионах где отсутствуют данные об уровне солнечной энергии, но есть иные данные метеорологических станций.

\section{Библиография :}

1. Измерение солнечного излучения в солнечной энергетике. [Электронный ресурc] http://www.kippzonen. com/Download/672/Solar-Energy-Guide-Russian (дата обращения: 13.02.2016).

2. Sozen A., Arcaklioglu E., Ozalp M., Kanit E.G. Use of Artificial neural network for mapping of solar potential in Turkey. Applied Energy, 2004, Vol. 77, No. 3, pp. 273-286.

3. Ahmed M., Ahmad F., Wasim M. Estimation of global and diffuse solar radiation for Hyderabad, Sindh, Pakistan, Journal of Basic and Applied Sciences, 2009, Vol. 5, No. 2, pp. 73-77. 
4. Мустафаев А.Г. Применение искусственных нейронных сетей для ранней диагностики заболевания сахарным диабетом // Кибернетика и программирование. - 2016. - № 2. - С.1-7.

DOI: 10.7256/2306-4196.2016.2.17904.URL: http://e-notabene.ru/kp/article_17904.html

5. Круглов В.В., Борисов В.В. Искусственные нейронные сети. Теория и практика. - М.: Горячая линия - Телеком, 2001. - 382 C.

6. Научно-прикладной справочник «Климат России». [Электронный ресурс] http://aisori.meteo.ru/ClspR (дата обращения: 14.02.2016).

7. Царегородцев В.Г. Оптимизация предобработки данных: константа Липшица обучающей выборки и свойства обученных нейронных сетей // Нейрокомпьютеры: разработка, применение. 2003, №7. - С.3-8.

8. Осовский С. Нейронные сети для обработки информации. - М.: Финансы и статистика, 2002. - 344 с.

9. Галушкин А. Нейронные сети. Основы теории. Горячая Линия - Телеком, 2012. - 496 с.

\section{References:}

1. Izmerenie solnechnogo izlucheniya v solnechnoi energetike. [Elektronnyi resurs] http://www.kippzonen.com/ Download/672/Solar-Energy-Guide-Russian (data obrashcheniya: 13.02.2016).

2. Sozen A., Arcaklioglu E., Ozalp M., Kanit E.G. Use of Artificial neural network for mapping of solar potential in Turkey. Applied Energy, 2004, Vol. 77, No. 3, pp. 273-286.

3. Ahmed M., Ahmad F., Wasim M. Estimation of global and diffuse solar radiation for Hyderabad, Sindh, Pakistan, Journal of Basic and Applied Sciences, 2009, Vol. 5, No. 2, pp. 73-77.

4. Mustafaev A.G. Primenenie iskusstvennykh neironnykh setei dlya rannei diagnostiki zabolevaniya sakharnym diabetom // Kibernetika i programmirovanie. — 2016. - № 2. - S.1-7. D0I: 10.7256/2306-4196.2016.2.17904. URL: http://e-notabene.ru/kp/article_17904.html

5. Kruglov V.V., Borisov V.V. Iskusstvennye neironnye seti. Teoriya i praktika. - M.: Goryachaya liniya - Telekom, 2001. - $382 \mathrm{~s}$.

6. Nauchno-prikladnoi spravochnik «Klimat Rossii». [Elektronnyi resurs] http://aisori.meteo.ru/ClspR (data obrashcheniya: 14.02.2016).

7. Tsaregorodtsev V.G. Optimizatsiya predobrabotki dannykh: konstanta Lipshitsa obuchayushchei vyborki i svoistva obuchennykh neironnykh setei // Neirokomp'yutery: razrabotka, primenenie. 2003, №7. - S.3-8.

8. Osovskii S. Neironnye seti dlya obrabotki informatsii. — M.: Finansy i statistika, 2002. - $344 \mathrm{~s}$.

9. Galushkin A. Neironnye seti. Osnovy teorii. Goryachaya Liniya - Telekom, 2012. - 496 s. 